\title{
Signal transducer and activator of transcription 6 as a target in colon cancer therapy (Review)
}

\author{
YAEL DELGADO-RAMIREZ ${ }^{1}$, VANEESA COLLY ${ }^{1,2}$, \\ GIOVANNI VILLANUEVA GONZALEZ ${ }^{2}$ and SONIA LEON-CABRERA ${ }^{1,2}$ \\ ${ }^{1}$ Laboratory of Oncoimmunology, Biomedical Research Unit; ${ }^{2}$ Medical School, Faculty of Superior \\ Studies Iztacala, National Autonomous University of Mexico, Tlalnepantla, CP 54090, Mexico
}

Received October 1, 2019; Accepted April 17, 2020

DOI: $10.3892 / \mathrm{ol} .2020 .11614$

\begin{abstract}
Signal transducer and activator of transcription 6 (STAT6) is a member of the STAT family of proteins that serve key roles in the initiation of tumorigenesis and malignant transformation. STAT6 is highly expressed in several types of cancer, including breast, pancreatic, prostate and colorectal cancer. STAT6 transduces signals in response to the binding of interleukin (IL)-4 and IL-13 to their receptors and regulates the expression of genes involved in the immune response, cell survival, tumor proliferation and metastasis. Patients with colorectal cancer exhibit high STAT6 activity in the colonic epithelium, and STAT6 expression is associated with lower survival rates, lymph node metastasis, changes in the epithelial barrier function and alterations in the inflammatory response. A number of studies investigating experimental models and cancer cell lines have revealed that STAT6 is associated with tumor growth and development, as well as with increased invasion and metastasis, suggesting that STAT6 inhibition may serve as a novel therapeutic strategy in colon cancer. The present review summarizes the evidence with regard to the implications of STAT6 in cancer biology and the direct and indirect effects on colon tumor transformation. Furthermore, the current treatment strategies targeting the IL-4/IL-13/STAT6 axis in colon cancer are discussed.
\end{abstract}

\section{Contents}

1. Introduction

2. Overview of the IL-4/IL-13/STAT6 signaling pathway

Correspondence to: Dr Sonia Leon-Cabrera, Laboratory of Oncoimmunology, Biomedical Research Unit, National Autonomous University of Mexico, Avenida De los Barrios 1, Los Reyes Iztacala, Tlalnepantla, CP 54090, Mexico

E-mail: soleonca@comunidad.unam.mx

Key words: colon cancer, signal transducer and activator of transcription 6, interleukin-4, interleukin-13, signaling, targeted therapy
3. STAT6 and its role in CRC

4. Targeting STAT6 for colon cancer therapy

5. Targeting IL-4, IL-13, IL-4R and IL-13R $\alpha 2$ in colon cancer therapy

6. Clinical trials targeting JAKs in colon cancer therapy

7. Conclusions

\section{Introduction}

Colorectal cancer (CRC) has the second highest cancerassociated mortality rate worldwide and is the third most commonly diagnosed neoplasm; a total of 881,000 CRC-associated mortalities and 1.8 million newly diagnosed cases were reported in 2018 (1). Sporadic CRC may be caused by a hereditary mutation, such as in patients with familial adenomatous polyposis with bi-allelic inactivation of the adenomatous polyposis coli (APC) gene or in patients with Lynch syndrome. The remaining cases exhibit activating mutations in components of the Wnt signaling pathway that promote malignant cell proliferation (2). Additionally, sustained inflammation in the intestine, such as in patients with inflammatory bowel disease (IBD), favors the development of colitis-associated cancer (CAC).

During chronic inflammation in the intestine, barrier dysfunction increases susceptibility to bacterial infection. Furthermore, mucosal inflammation results in the accumulation of mutations that can result in the development of colon cancer. In CAC, inflammatory cytokines are persistently present in the intestinal tissue, and in patients with sporadic cancer, the administration of anti-inflammatory drugs can prevent or delay the disease, suggesting that inflammatory processes are involved in the initiation of tumorigenesis (3).

Cytokines serve as a means of communication among immune, cancer and non-transformed stromal cells in the tumor microenvironment. The signal transducer and activator of transcription (STAT) family of proteins serve an important role in the initiation of malignant transformation and in tumor establishment, and have been widely studied in experimental models and in patients with cancer (4). Upon cytokine binding to its receptor, Janus kinases (JAKs) mediate STAT phosphorylation. In the nucleus, STAT binds to specific DNA sequences that result in the transcription of target genes (5). Interleukin 
(IL)-6, IL-10 and IL-23 signaling is mediated by JAK1 and recruits STAT3. Conversely, STAT6 functions as a transcription factor in the nucleus in response to IL-4 and IL-13 receptor binding after the activation of JAK1 and JAK3 (5). STAT6 orchestrates numerous processes beyond immune response, including cancer cell proliferation, apoptosis resistance, metastasis, epithelial cell function, chromatin compaction, and DNA damage and repair (Table I). STAT6 signaling is frequently activated in malignant cells and regulates several genes crucial for the immune response, inflammation and proliferation (5). Persistent activation of STAT6 in different types of cancer results in proliferation, survival and metastasis, as well as in decreased antitumor immunity. STAT6 polymorphisms have been identified in a subgroup of Malaysian patients suffering from Crohn's disease (CD), as well as in a patient cohort in Germany (6-8), demonstrating the importance of this gene in inflammatory processes in the colon.

Despite the advances in the diagnosis and treatment of $\mathrm{CRC}$, the mortality rates remain high. Therefore, there is a requirement for the development of alternative therapeutic strategies for this disease. Interfering with the activity of STAT6 could be a potential strategy to target the action of IL-4 and IL-13, and the activation of other signaling pathways involved in tumorigenesis.

The present review describes the direct and indirect effects of STAT6 on colon tumor transformation and discusses the role of STAT6 in mucosal biology. Additionally, the current treatment strategies that target the IL-4/IL-13/STAT6 axis in colon cancer are summarized.

\section{Overview of the IL-4/IL-13/STAT6 signaling pathway}

STAT6 serves important roles in signal transduction throughout the cytoplasm and is a transcription factor in the nucleus. Cytokines and growth factors bind to their cognate receptors and activate the JAK family. Once activated, JAKs phosphorylate docking sites in the SH2 domain of STAT molecules. After phosphorylation, STATs form homodimers or heterodimers, which are transported into the nucleus where they regulate the expression of genes (5).

STAT6 activation occurs by IL-4 or IL-13 binding to their receptors. Currently, three types of receptors have been described. The IL-4 type I receptor is composed of the IL-4 receptor $\alpha$ (IL-4R $\alpha$ ) chain and the common $\gamma$ chain $(\gamma \mathrm{c})$. The IL-4R $\alpha$ chain has a high affinity for IL-4, while the $\gamma c$ is a component of receptors that bind IL-2 and IL-9. IL-4 binds to the IL-4R $\alpha$ chain, resulting in dimerization with the $\gamma \mathrm{c}$ chain and receptor activation (9). However, in human colon carcinoma cells, it has been reported that the IL-4R $\alpha$ chain does not bind with the $\gamma \mathrm{c}(10)$.

The type II receptor is composed of the IL-4R $\alpha$ and IL-13 receptor $\alpha$ variant 1 (IL-13R $\alpha 1$ ) chains, and is able to bind to both IL-4 and IL-13. IL-13 binds to the monomer IL-13R $\alpha 1$ with low affinity, and requires heterodimerization with IL-4R $\alpha$ to form a high-affinity bond. This complex recruits JAKs and leads to the downstream activation of STAT6 (9).

Additionally, IL-13 can bind to the IL-13 receptor $\alpha$ variant 2 (IL-13R $\alpha 2$ ) chain; this receptor is a monomer that binds IL-13 with higher affinity than IL-13R $\alpha 1$ and is expressed in T cells, B cells and endothelial cells, among others, where it has been demonstrated to promote tumorigenesis (11). IL-13R $\alpha 2$ is considered as a decoy receptor, as it is able to bind IL-13 and prevent it from binding to IL-13R $\alpha 1$ (11).

After stimulation of the IL-4R $\alpha$ receptor, JAK1-3 and tyrosine kinase 2 (Tyk2) are phosphorylated in the cytoplasmic tails of the receptor. Once active, JAKs phosphorylate the tyrosine residues Y575, Y603 and Y631 on the receptor, generating docking sites for STAT6. When monomers of STAT6 become phosphorylated on tyrosine Y641, the C-terminal of the SH2 domain usually forms homodimers (12) that are translocated to the nucleus, where they can bind DNA and activate or repress target genes. Therefore, the activity of STAT6 depends on tyrosine phosphorylation. However, the proximity of subunits may influence heterodimer formation. The stimulation of B cells with interferon (IFN) type 1 promotes the generation of STAT2:STAT6 heterodimers (13).

Proper regulation of STAT6 signaling is crucial, and specific molecules have been identified to regulate this signaling pathway. Suppressor of cytokine signaling (SOCS) proteins, particularly SOCS1, repress the activation of JAK1/3 and STAT6, and affect IL-4-induced proliferation (14). STAT6 signaling induces SOCS1 expression in a negative feedback loop, inhibiting the expression of STAT6-responsive genes (14). Additionally, the IL-4/STAT6 signaling pathway induces SOCS3 expression in intestinal epithelial cells. Increased SOCS3 levels in patients with ulcerative colitis (UC) are indicative of disease exacerbation (15). STAT6 activation is responsible for SOCS3 induction and affects the regulation of STAT1 and STAT3 signaling (15).

Protein tyrosine phosphatase (SHP1) negatively regulates IL-4/IL-13 signaling and STAT6 gene induction (16). Studies investigating the overexpression of SHP1 demonstrated a decrease in IL-4/IL-13 signaling, suggesting that tyrosine phosphorylation of STAT6 is regulated by SHP1 (16). Protein tyrosine phosphatase 1B (PTP1B) has been demonstrated to dephosphorylate cytoplasmic and nuclear STAT6, and attenuate its signaling. In diffuse large B-cell lymphomas, PTP1B contributes to STAT6 dephosphorylation, thereby enhancing tumorigenesis and inflammatory processes (17). Additionally, STAT6 function is regulated by methylation. When STAT6 is methylated on arginine at the N-terminus, decreased phosphorylation, nuclear translocation and DNA-binding activity are observed in response to IL-4 (18).

Several target genes of STAT6 have been identified and characterized. The STAT6 signal transduction pathway serves an important role in mediating the biological functions of IL-4 and IL-13 in processes associated with Th2 immune responses. During allergic reactions, STAT6 mediates T helper 2 (Th2) cells and eosinophil recruitment within sites of allergic inflammation, and is involved in immunoglobulin (Ig) class switching to produce IgE (5).

However, abnormal STAT6 activation may contribute to the pathology of cancer by increasing the expression of proteins involved in proliferation, migration and invasion. The IL-4/STAT6 signaling pathway increases nuclear survivin in CRC stem cells, allowing them to evade cell death (19). Platelet-derived growth factors (PDGFs) are secreted by human vascular endothelial cells, epithelial cells and fibroblasts. PDGF is a survival factor that inhibits apoptosis, promotes proliferation and stimulates mesenchymal cell proliferation 


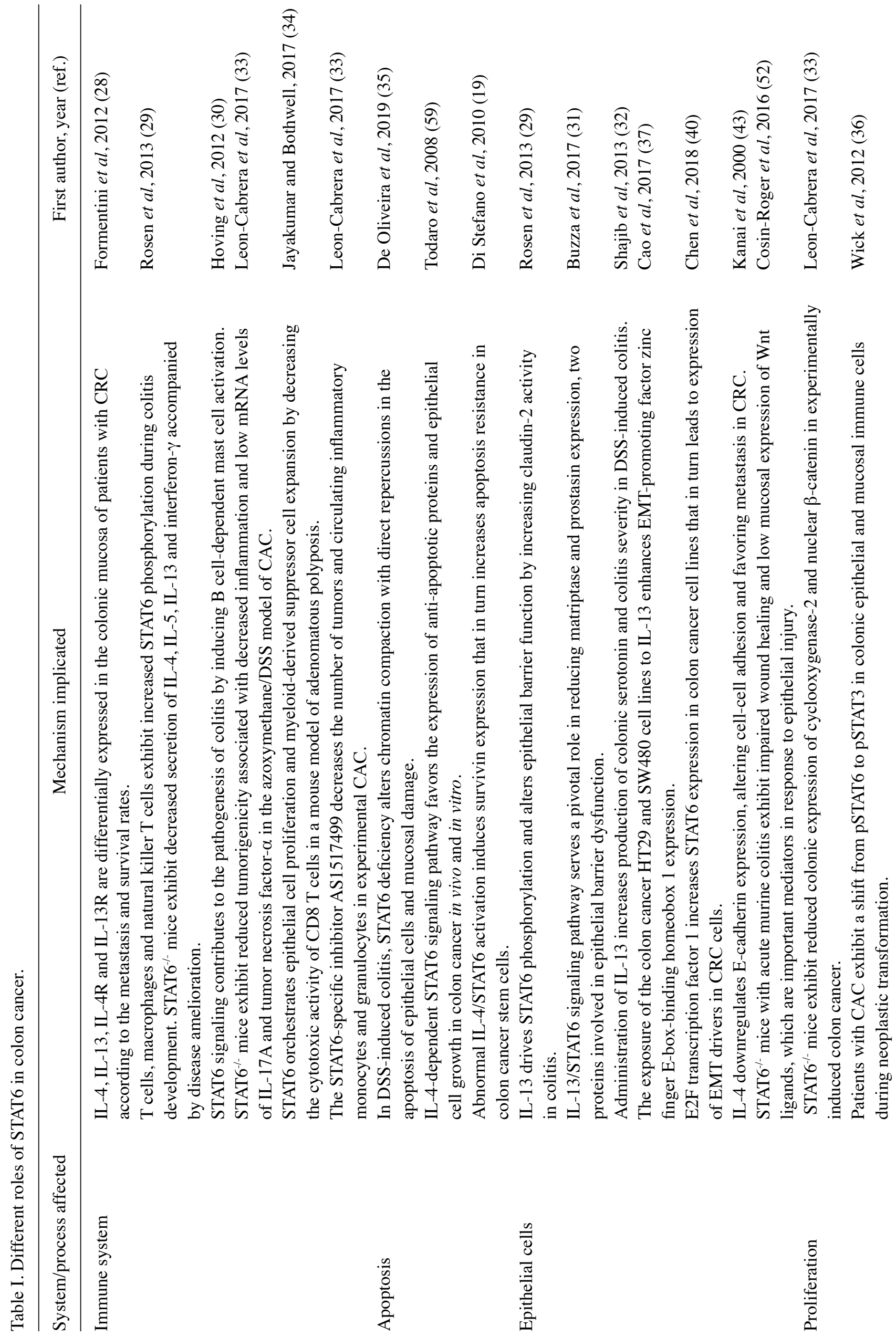


and migration (20). A previous study suggested that PDGF and STAT6 have related functions. PDGF is able to activate STAT6 in fibroblasts to promote their proliferation (21). The IL-13/STAT6 signaling pathway upregulates PDGF mRNA expression levels in lung fibroblasts, and STAT6 is required for PDGF-A and PDGF-C gene expression, with important implications in tumor development (22). Therefore, STAT6 is a key regulatory molecule with tumor proliferating functions that may aid the identification of molecular targets for the treatment of colon cancer.

\section{STAT6 and its role in CRC}

Clinically detectable IBD increases the risk of CAC; patients with UC have an increased risk (2.4-fold) of developing CAC (23), while patients with CD have an increased risk (2.59-fold) of developing CAC in the next two decades, compared with the general population (24).

Expression of the constitutively activated IL-4/IL-13/STAT6 axis during UC, $\mathrm{CD}$ and in colon cancer tissues suggests that this pathway may contribute to the underlying pathology of these diseases. In a previous study, immunohistochemistry analyses were performed to detect phosphorylated STAT6 (pSTAT6) in colonic tissues of pediatric subjects with UC or $\mathrm{CD}$, and it was demonstrated that nuclear pSTAT6 was significantly upregulated in the colonic epithelium (25). Furthermore, STAT6 was significantly upregulated in neoplastic tissues of patients with CRC, and its expression was associated with lower survival rates and with lymph node metastasis (26). Gene expression levels of IL-4, IL-5 and IL-13 were significantly upregulated in tumors compared with those in normal tissues of patients with CRC; however, no apparent effect on clinical outcome was observed (27). By contrast, a study performed by Formentini et al (28) revealed that IL-4, IL-13, IL-4R and IL-13R were expressed in CRC specimens, and that high expression levels of IL-4, IL-4R and IL-13R were associated with a lower frequency of lymph node metastasis, while IL-13 expression was associated with a better overall survival rate.

IL-13-induced activation of STAT6 has been implicated in mediating the host inflammatory cascade in UC. A mouse model of oxazolone-induced UC demonstrated that IL-13 is responsible for inducing the expression of the pore-forming tight junction protein claudin-2, increasing epithelial barrier permeability and compromising gastrointestinal barrier function (29). In addition, epithelial cells, T cells, macrophages and natural killer T cells exhibit increased STAT6 phosphorylation during colitis development (29). When colitis was induced in STAT6-deficient mice, decreased pathology, claudin-2 expression, and secretion of IL-4, IL-5, IL-13 and IFN- $\gamma$ were observed (29). CD4 ${ }^{+}$Th2 cells produce IL-13, which favors IgE production by B cells, and induce mast cell activation, suggesting that STAT6 contributes to the pathogenesis of colitis (30). Therefore, IL-13 seems to drive STAT6 phosphorylation, alter epithelial barrier function, regulate Th2 cytokine production and mediate the inflammatory response to colitis.

The IL-13/STAT6 signaling pathway may be detrimental for intestinal epithelial cell function. The downregulation of matriptase and prostasin, two membrane-anchored serine proteases important for the epithelial barrier development and for homeostasis, was observed in a dextran sulfate sodium 


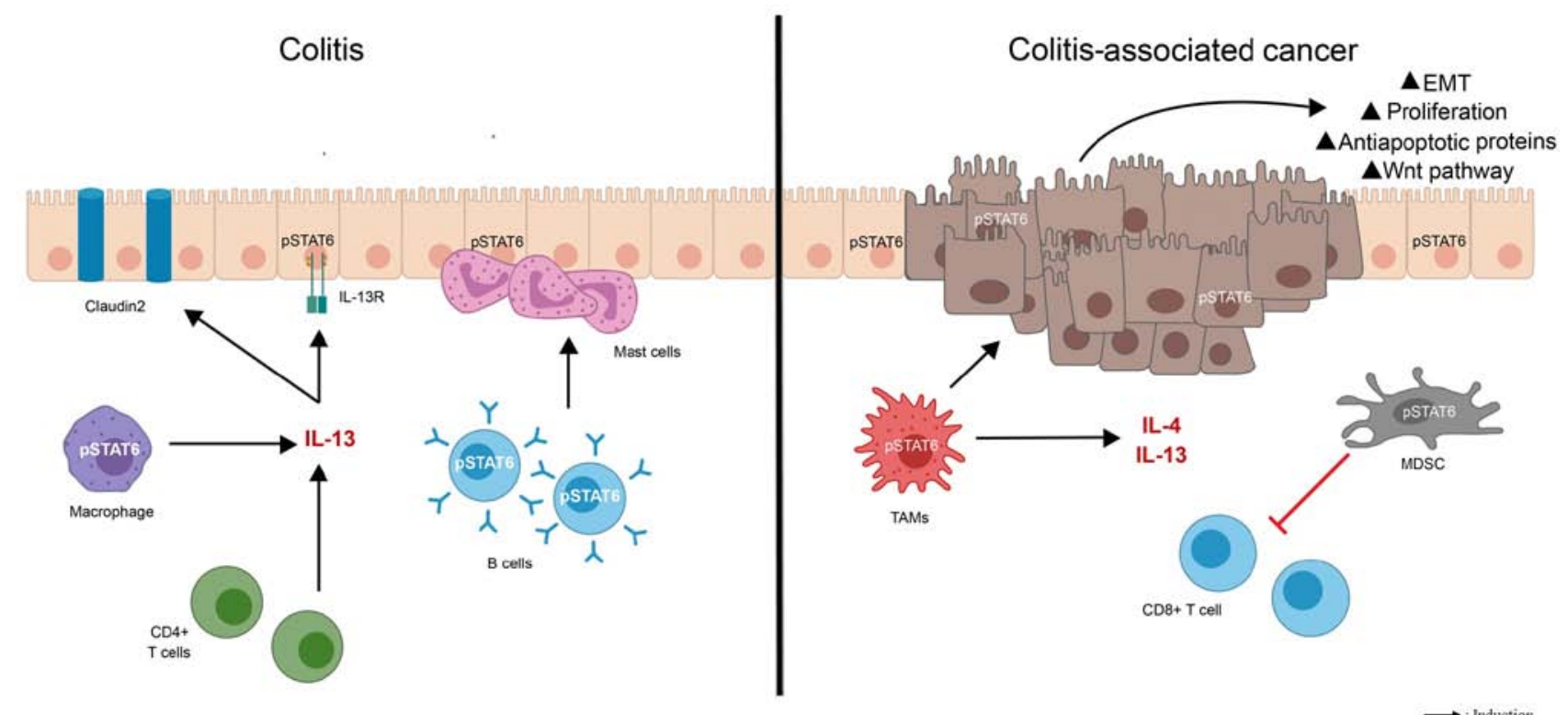

$\longrightarrow$ : Induction

Figure 1. STAT6 modulates pro-colitis and pro-cancer responses. The IL-13/STAT6 signaling pathway appears to be detrimental to the function of intestinal epithelial cells in subjects with ulcerative colitis or Crohn's disease, in which nuclear pSTAT6 is significantly increased in the colonic epithelium. IL-13 signaling alters the expression of proteins, including the pore-forming tight junction protein claudin-2, matriptase, prostasin and serotonin, involved in epithelial barrier permeability and gastrointestinal barrier function via STAT6. In addition, IL-13 produced by CD4+ T helper 2 cells favors STAT6 phosphorylation in mast cells, B cells and macrophages during the development of colitis, suggesting that STAT6 signaling in immune cells favors the progression of the disease. In the tumor microenvironment, STAT6 coordinates both polyp cell proliferation and myeloid-derived suppressor cell expansion. Furthermore, the IL-4/STAT6 signaling pathway induces the expression of anti-apoptotic proteins and the growth of epithelial colon cancer cells. STAT6 activation prompts the differentiation of infiltrating macrophages into a tumor-associated phenotype with pro-tumoral functions; these M2 macrophages promote mucosal repair through the activation of the Wnt signaling pathway. However, mutations that cause aberrant Wnt signaling are responsible for polyp development in the small intestine and colon. Additionally, the exposure to IL-13 enhances the expression of epithelial-to-mesenchymal transition-promoting factors, resulting in a decrease in adhesion, migration, invasion and metastatic colonization. pSTAT6, phosphorylated signal transducer and activator of transcription 6; IL-R, interleukin receptor; TAMs, tumor-associated macrophages; MDSC, myeloid-derived suppressor cell.

(DSS)-induced model of colitis in mice and in colonic tissues from human subjects with active UC and CD (31). When STAT6 was inhibited by suberoylanilide hydroxamic acid (SAHA), the expression levels of matriptase and prostasin were restored and barrier dysfunction was decreased, implicating STAT6 signaling in the loss of the barrier-protective protease pathway (31). Enterochromaffin cells, which are responsible for synthesizing serotonin to increase epithelial cell secretion, express IL-13R (32). In a model of DSS-induced colitis, the administration of IL-13 increased colonic serotonin production and exacerbated the severity of colitis (32). It is unclear whether these effects are unique to colitis or increase the risk of developing CRC. Previous studies have revealed that STAT6 serves important roles in the early steps of colitis-associated carcinogenesis and that it modulates inflammatory responses, as well as controlling cell recruitment and proliferation $(33,34)$. CAC induction in STAT6-deficient mice $\left(\mathrm{STAT6}^{-/}\right.$) in a azoxymethane/DSS model resulted in reduced tumorigenicity, associated with reduced inflammation, decreased concentrations of cyclooxygenase-2 (COX2) and nuclear $\beta$-catenin protein in the colon, and decreased mRNA expression levels of cytokines IL-17A and tumor necrosis factor- $\alpha$ (TNF- $\alpha$ ) (33). In addition, the number of circulating inflammatory monocytes and granulocytes was decreased in STAT6 ${ }^{-/-}$mice (33). Furthermore, STAT6 deletion in the $\mathrm{Apc}^{\mathrm{Min} /+}$ mouse model reduced the incidence of polyps in the small intestine and decreased the proliferation of polyp epithelial cells; this effect was attributed to an expansion of myeloid-derived suppressor cells (MDSCs), implying regulation of the antitumor T-cell response in a STAT6-dependent manner (34). Therefore, STAT6 may serve a broad role in coordinating both polyp cell proliferation and MDSC expansion. By contrast, Oliveira et al (35) reported that STAT6-deficient mice are more susceptible to mucosal damage during DSS-induced colitis, and exhibit enhanced intestinal epithelial cell apoptosis, tissue injury and inflammatory responses. Chromatin condensation in intestinal epithelial cells is affected by STAT6 signaling and may protect cells from apoptosis and severe tissue damage (35). However, the role of STAT6 during different stages of colitis and tumor cell proliferation has not been fully elucidated.

The activation and function of STAT6 in colitis along the continuum of inactive disease to CAC are dynamic. In a previous study, immunohistochemistry to detect pSTAT1, pSTAT6 and pSTAT3 in colonic epithelial and mucosal immune cells in patients with UC and CAC was performed. A shift from predominant STAT6 activation in immune cells to STAT3 activation accompanied the onset of dysplasia with a concomitant increase in epithelial cell STAT3 activation in low- and high-grade tumors. STAT6 expression was frequently detected in normal tissues, but not in CAC tissues (36). The decrease in pSTAT6 expression in both immune cells and intestinal epithelial cells indicates that STAT6 signaling may be detrimental in the transition from colitis to cancer. Nevertheless, abnormal STAT6 signaling has been implicated in pro-metastatic processes, including 


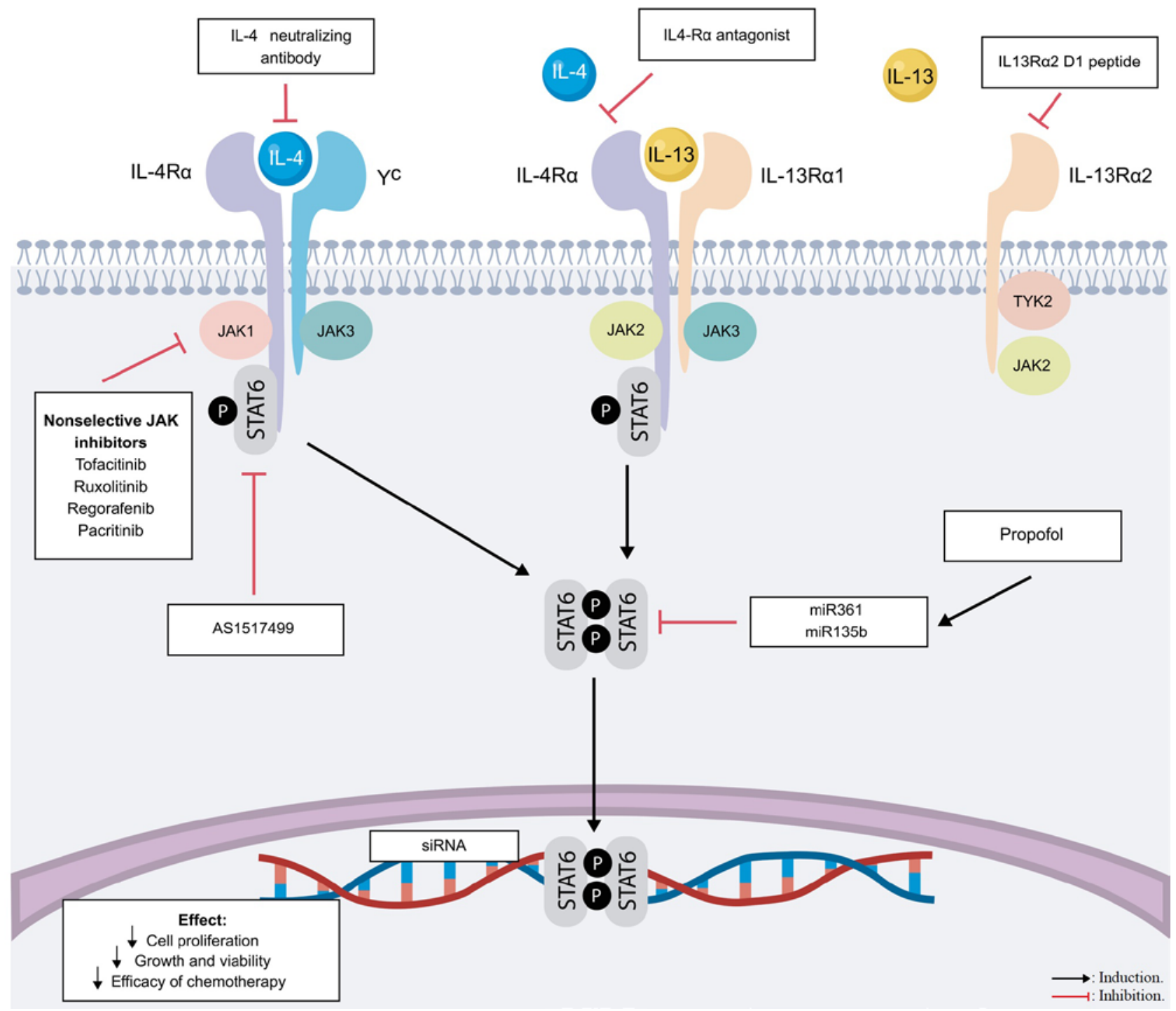

Figure 2. Targeting the IL-4/IL-13/STAT6 signaling pathway for cancer therapy. Upon cytokine-receptor binding, JAK1, JAK2 or tyrosine kinase 2 are recruited and activated, inducing the phosphorylation of STAT6. STAT6 homodimers translocate to the nucleus and bind DNA sequences at genes involved in apoptosis, proliferation and the immune response. Various drug targets have been evaluated to block IL-4, IL-4R, IL-13R $\alpha 1$ or STAT6. Points of potential therapeutic inhibition of the pathway are indicated. STAT6, signal transducer and activator of transcription 6; IL-R, interleukin receptor; JAK, Janus kinase; miR, microRNA; siRNA, small interfering RNA; $\gamma \mathrm{c}, \gamma$ chain.

the proliferation and survival of colon cancer cells (37-39). During the epithelial-to-mesenchymal transition (EMT), epithelial cells transform into aggressive phenotypes with enhanced migratory capacity and invasiveness. Exposure to IL-13 enhances the expression of the EMT-promoting factor zinc finger E-box binding homeobox 1 (ZEB1) in the colon cancer HT29 and SW480 cell lines (37). When STAT6 is blocked or knocked down, the IL-13-induced EMT and ZEB1 induction in CRC cells is reversed (37). Additionally, a positive association between IL-13R $\alpha 1$ and ZEB1 at the mRNA level has been observed in human CRC samples, demonstrating that the IL-13/STAT6 signaling pathway serves a critical role in promoting EMT and the aggressiveness of CRC (37). $11 \beta$-hydroxysteroid dehydrogenase type II (11ßHSD2) is a key enzyme induced in an IL-13R $\alpha 2$-dependent manner that promotes the expression of Akt and COX2; upon inhibiting $11 \beta H S D 2$, liver metastasis is decreased, suggesting that IL-13 may regulate malignancy via $11 \beta \mathrm{HSD} 2$ during CRC (38). In addition, highly metastatic CRC cells express high levels of IL-13R $\alpha 2$ (39). IL-13R $\alpha 2$ silencing results in a decrease in adhesion, migration, invasion and metastatic colonization. In a previous study, the upregulation of IL13R $\alpha 2$ expression in $66 \%$ of tumor samples from patients with colon cancer was associated with late stages of progression (metastasis in lymph nodes or liver) and a poor outcome in patients with CRC. Highly metastatic CRC cells exhibit activation of PI3K, Akt and SRC proto-oncogene non-receptor tyrosine kinase in response to IL-13, supporting the role of the IL-13/IL-13R/STAT6 signaling pathway in CRC cell invasion and metastasis (39).

Additionally, STAT6 phosphorylation is induced by IL-4. E2F transcription factor 1 (E2F1), a critical transcription factor for CRC development, increases STAT6 expression in colon cancer cells and increases their susceptibility to IL-4 stimulation. E2F1 acts as an enhancer of the IL-4/STAT6 signaling pathway and increases the expression of EMT drivers in CRC cells (40). IL-4 is able to induce the expression 
of anti-apoptotic proteins and the growth of epithelial colon cancer cells in vivo and in vitro. IL-4 neutralization sensitizes colon carcinoma cells to chemotherapy and to death by the TNF-related apoptosis-inducing ligand (41). Abnormal IL-4/STAT6 activation induces the expression of survivin, a protein with an important role in apoptosis resistance in colon cancer stem cells; the use of leflunomide, a STAT6 inhibitor, has been shown to decrease survivin expression and localization, thereby reducing its anti-apoptotic effect (19). An increase in IL-4 expression contributes to an oxidant milieu, thereby increasing the expression of NADPH oxidase 1 and reactive oxygen species, resulting in DNA damage and the neoplastic transformation of colon cells (42). Further evidence indicates that IL-4 is implicated in the inhibition of colon cancer cell-cell adhesions, acting as a negative regulator in the expression of E-cadherin, a key component of adherent junctions, and in maintaining epithelial cell adhesion and decreasing invasiveness (43).

Increasing evidence demonstrates that STAT6 serves an important role in the regulation of tumor immunity. The use of the STAT6-specific inhibitor AS1517499 has been shown to decrease colonic tumor load and the number of circulating inflammatory monocytes and granulocytes in experimental models of CAC (33). The reduced number of the aforementioned cells may be associated with decreased macrophage infiltration and reduced tumor growth. IL-4/IL-13 cytokines, via STAT6 activation, induce the differentiation of infiltrating macrophages to an M2 phenotype with pro-tumoral functions. The pharmacological inhibition of STAT6 with AS1517499 was shown to attenuate tumor growth and liver metastasis in an orthotopic 4T1 mammary carcinoma mouse model (44). This result was associated with a decrease in tumor-associated macrophages displaying the M2 phenotype, suggesting that IL-4 blockade inhibits tumor angiogenesis and growth by reprograming macrophages to a less aggressive phenotype (45). In addition, the deletion of the STAT6 gene was demonstrated to improve antitumor immunity in the same model via a type 1 $\mathrm{CD} 4^{+}$response (46).

Autocrine production of IL-4 by colon cancer cells protects against apoptosis and chemotherapy-induced cell death (47-50). It was found that IL-4 was highly expressed in cancer-initiating cells obtained from patients with CRC and was associated with low immunogenic profiles; blocking IL-4 increased Th1-type CD8 ${ }^{+} \mathrm{T}$ cells responses in vitro (51). Previous studies also revealed that STAT6 maintains mucosal homeostasis by sustaining myeloid cells. STAT6 ${ }^{-/}$mice with acute murine colitis exhibited delayed wound healing and decreased mucosal expression levels of Wnt ligands, which are important responses to epithelial injury (52). The administration of M2 macrophages to STAT6 ${ }^{-/}$mice promoted mucosal repair through the activation of the Wnt signaling pathway; however, mutations that cause aberrant Wnt signaling are responsible for polyp development in the small intestine and colon (52). In the $\mathrm{APC}^{\mathrm{min} /+}$ mouse model, which recapitulates the disease observed in patients with familial APC, STAT6 promoted the expansion of MDSCs and decreased the cytotoxicity of CD8 $\mathrm{T}$ cells, contributing to intestinal tumorigenesis (34). The identification of the direct functions of STAT6 on immunosurveillance and epithelial cell homeostasis may serve as the basis for the development of novel immunotherapy strategies.
The aforementioned results demonstrate that the IL-4/IL-13/STAT6 axis may serve a role in inflammation, tumor cell proliferation, cancer cell survival and metastasis, and that the interference of its effects in colitis or in tumor cells may present a novel strategy for the treatment of CRC (Fig. 1; Table I).

\section{Targeting STAT6 for colon cancer therapy}

To the best of our knowledge, the use of specific STAT6 inhibitors in preclinical and clinical studies for colon cancer has not been reported. However, in experimental models and cancer cell lines, STAT6 inhibition decreases tumor cell proliferation, survival, adhesion, invasion and metastasis, suggesting that STAT6 inhibition may serve as a therapeutic target in colon cancer.

The use of the STAT6-specific inhibitor AS1517499 reduced tumor growth and signs of the disease in mice with CRC $(33,53)$. In vivo, STAT6 inhibition reduced colonic tumor load and colon tissue damage, corresponding with a decrease in STAT6 phosphorylation in the intestine (33). In an orthotopic 4T1 mammary carcinoma mouse model, the use of AS1517499 attenuated tumor growth and early liver metastasis (44). Similarly, in primary epithelial cells from patients with prostate cancer, exposure to AS1517499 decreased IL-4-induced colony formation (54). Upregulation of microRNA (miRNA/miR)-361 and miR-135b is associated with a decrease in STAT6 expression (55). The common intravenous anesthetic agent propofol has been associated with a reduction in tumor-associated inflammation and with the ability to induce miRNAs to suppress STAT6 expression (55). The treatment of the CRC SW480 and RKO cell lines with propofol was shown to increase the expression levels of miR-135b and miR-361, which are STAT6-targeting miRNAs, and decrease cell proliferation and migration, suggesting that propofol interferes with the IL-13/STAT6 signaling pathway (55). Nevertheless, the in vivo effect of propofol requires further investigation. A preclinical model utilizing small interfering RNA (siRNA) to specifically suppress STAT6 expression in lung epithelial cells was reported (56); the study stated that the intranasal application of STAT6 siRNAs attenuated allergic airway inflammation, demonstrating that STAT6 may be targeted in specific tissues. Therefore, further studies evaluating the possibility of suppressing STAT6 signaling in the colonic epithelium are required. The use of STAT6 inhibitors results in the inhibition of type I and type II IL-4Rs, and therefore, the negative implications for normal immune functions should be determined.

\section{Targeting IL-4, IL-13, IL-4R and IL-13R $\alpha 2$ in colon cancer therapy}

IL-4 and IL-13 bind to their receptors and activate the JAK/STAT6 signaling pathway. Therefore, the use of molecules that can block binding or impair the signaling of these cytokines to prevent STAT6 activation may be clinically relevant. Previous studies have revealed that blocking IL-4 and IL-13, as well as their receptors, has different consequences on tumor development (Fig. 2). 
The synthetic IL-13R $\alpha 2 \mathrm{D} 1$ peptide inhibits IL-13-mediated STAT6 activation through IL-13R $\alpha 1$, and blocks IL-13 binding to IL-13R $\alpha 2$, decreasing IL-13 signaling; the administration of IL-13R $\alpha 2$ D1 peptide was previously shown to repress tumor growth, invasion and proliferation in a metastatic CRC mouse model (57).

The direct administration of anti-IL-4 antibody in combination with chemotherapy suppresses the growth and viability of the Caco cell line; this effect is associated with a decrease in the expression levels of the CRC stem cell marker CD133, suggesting that anti-IL-4 therapy may enhance the efficacy of chemotherapy regimens (58). When conventional drugs, such as 5-fluorouracil or oxaliplatin, are combined with an IL-4 neutralizing antibody or an inhibitory form of IL-4, the efficacy of chemotherapy is enhanced in both mature cancer cells and cancer-stem like cells (59). Similarly, it has been shown that when IL-4 and IL-13 responses are inhibited by an IL-4R $\alpha$ antagonist in nude mice injected with colon cancer spheroids, the tumor response to chemotherapeutic drugs is enhanced (60). The increased production of IL-4 in cancer cells may favor a death-resistant phenotype, and limiting the production and/or signaling of IL-4 may be an alternative approach for treatment-resistant cells.

The antitumor effect of doxorubicin has been widely reported (61). In a previous study, the liposomal form of doxorubicin was conjugated with a ligand of the atherosclerotic plaque-specific peptide-1 (AP1), a peptide characterized by its ability to bind IL-4R; Yang et al (62) proposed that AP1-conjugated liposomal doxorubicin exhibits an increased and selective cytotoxic effect on CRC cells, and has potential as a targeted anticancer therapy. Collectively, the aforementioned studies demonstrated that the inhibition of IL-4, IL-4R, IL-13R $\alpha 1$ and IL-13R $\alpha 2$ may be beneficial for colon cancer.

\section{Clinical trials targeting JAKs in colon cancer therapy}

It is well known that IL-4R, $\gamma \mathrm{c}$ and IL-13R $\alpha 1$ activate JAK1/2 and Tyk2. STAT6 is phosphorylated following JAK activation and forms homodimers that translocate to the nucleus. JAK molecules have been targeted therapeutically to treat rheumatoid arthritis, psoriasis and IBD $(63,64)$. The inhibition of the JAK/STAT inflammatory pathway may be an alternative for the treatment of CRC. However, to the best of our knowledge, JAK inhibitors have not been approved by the Food and Drug Administration (FDA) for the treatment of colon cancer.

In addition to activating STAT6, JAKs mediate a number of cytokine receptor responses (3). While several cytokines are involved in inflammatory responses that may promote cancer development, other cytokines regulate processes involved in mucosal healing, barrier function and immunosurveillance (3-5). For example, the inhibition of JAK1 may alter the signaling of IL-2, IL-7, IL-9, IL-6 and IL-10 (4). Changes in IL-10, an important anti-inflammatory cytokine, may affect intestinal homeostasis. In addition, the function of $\mathrm{T}$ cells and natural killer cells may be altered as a result of changes in cytokine signaling $(3,4)$. The inhibition of JAKs in response to IL-4 mediates alterations in Akt, ERK and mTOR, which are involved in several processes (12). Therefore, it is difficult to discern and control the effect of JAK inhibition, particularly in cancer development, where the tumor and stromal cells are actively interacting through cytokines. Consequently, the use of JAK inhibitors for the treatment of colon cancer is not widespread. Ruxolitinib, an oral selective inhibitor of JAK1/2, is approved by the FDA for use in myelofibrosis (65). Ruxolitinib was tested in a phase 2 study in combination with regorafenib, an oral multi-targeted kinase inhibitor, in patients with advanced and metastatic adenocarcinoma of the colon or rectum (66). However, there was no significant difference in the overall survival rate or progression-free survival rate between the regorafenib + placebo vs. regorafenib + ruxolitinib groups (66). Nevertheless, the treatment was administered in the advanced stages of tumor development, and the trial was terminated early per sponsor decision; therefore, further investigation is required, particularly during early stages of CRC.

Pacritinib, an oral inhibitor of JAKs and other kinases, was administrated to patients with metastatic CRC; however, the study did not produce conclusive results as the trial was discontinued prior to completion (64). Additionally, the use of tofacitinib, a JAK $1 / 3$ inhibitor widely used for the treatment of rheumatoid arthritis, was found to be associated with an increase in lung metastasis accompanied with a decrease in natural killer cell number in a mouse model of colon cancer (67). Therefore, the development of selective tissue-specific inhibitors may overcome these types of complications.

\section{Conclusions}

Several studies have revealed that the IL-4/IL-13/STAT6 axis contributes to the pathology of IBD and colon cancer. Nuclear pSTAT6 is significantly increased in colonic epithelium and in neoplastic tissues obtained from patients with CRC, and its expression is associated with lower survival rates. Additionally, STAT6 seems to regulate mechanisms that promote the proliferation, survival, invasion and metastasis of tumor cells, as well as the suppression of antitumor immunity. Inhibiting STAT6 signaling in experimental models and cancer cell lines has resulted in a decrease in cancer-associated processes. Therefore, STAT6 may serve as a potential target in the treatment of colon cancer. However, to the best of our knowledge, STAT6 inhibitors in preclinical and clinical studies for colon cancer have not been reported. The indirect inhibition of the STAT6 signaling pathway through IL-4, IL-13 or JAKs may inhibit carcinogenesis, but it may also result in side effects as a consequence of alterations in the immune response. Therefore, preclinical and clinical studies investigating specific STAT6 inhibitors with a targeted organ distribution are required to evaluate the potential of STAT6 as a target for colon cancer therapy.

\section{Acknowledgements}

Not applicable.

\section{Funding}

The present study was supported by grants from Support Program for Research Projects and Technological Innovation (PAPIIT)(grant no. IA204218) and National Council for Science and Technology (CONACYT)(grant no. A1-S23944). 


\section{Availability of data and materials}

Not applicable.

\section{Authors' contributions}

YDR and SLC contributed to the conception of the study, writing the manuscript and performing the literature search. VC and GVG collected and analyzed data. All authors read and approved the final manuscript.

\section{Ethics approval and consent to participate}

Not applicable.

\section{Patient consent for publication}

Not applicable.

\section{Competing interests}

The authors declare that they have no competing interests.

\section{Authors' information}

YDR is a doctoral student from the Biomedical Sciences Doctorate Program, at the National University of Mexico and received a National Council for Science and Technology fellowship (606590).

\section{References}

1. Bray F, Ferlay J, Soerjomataram I, Siegel RL, Torre LA and Jemal A: Global cancer statistics 2018: GLOBOCAN estimates of incidence and mortality worldwide for 36 cancers in 185 countries. CA Cancer J Clin 68: 394-424, 2018.

2. Kuipers EJ, Grady WM, Lieberman D, Seufferlein T, Sung JJ, Boelens PG, van de Velde CJ and Watanabe T: Colorectal cancer. Nat Rev Dis Primers 1: 15065, 2015.

3. West NR, McCuaig S, Franchini F and Powrie F: Emerging cytokine networks in colorectal cancer. Nat Rev Immunol 15: 615-629, 2015.

4. Yu H, Pardoll D and Jove R: STATs in cancer inflammation and immunity: A leading role for STAT3. Nat Rev Cancer 9: 798-809, 2009.

5. Hebenstreit D, Wirnsberger G, Horejs-Hoeck J and Duschl A: Signaling mechanisms, interaction partners, and target genes of STAT6. Cytokine Growth Factor Rev 17: 173-188, 2006 .

6. Chua KH, Ng JG, Ng CC, Hilmi I, Goh KL and Kee BP: Association of NOD1, CXCL16, STAT6 and TLR4 gene polymorphisms with Malaysian patients with Crohn's disease. Peerj 4: e1843, 2016

7. Klein W, Tromm A, Folwaczny C, Hagedorn M, Duerig N, Epplen J, Schmiegel W and Griga T: The G2964A polymorphism of the STAT6 gene in inflammatory bowel disease. Dig Liver Dis 37: 159-161, 2005

8. Xia B, Crusius JB, Wu J, Zwiers A, van Bodegraven AA and Pena AS: Signal transducer and activator of transcription 6 gene G2964A polymorphism and inflammatory bowel disease. Clin Exp Immunol 131: 446-450, 2003.

9. Mueller TD, Zhang JL, Sebald W and Duschl A: Structure, binding, and antagonists in the IL-4/IL-13 receptor system. Biochim Biophys Acta 1592: 237-250, 2002.

10. Murata T, Noguchi PD and Puri RK: Receptors for interleukin (IL)-4 do not associate with the common gamma chain, and IL-4 induces the phosphorylation of JAK 2 tyrosine kinase in human colon carcinoma cells. J Biol Chem 270: 30829-30836, 1995.
11. Sengupta S, Thaci B, Crawford AC and Sampath P: Interleukin-13 receptor alpha 2-targeted glioblastoma immunotherapy. Biomed Res Int 2014: 952128, 2014.

12. Mikita T, Campbell D, Wu P, Williamson K and Schindler U: Requirements for interleukin-4-induced gene expression and functional characterization of Stat6. Mol Cell Biol 16: 5811-5820, 1996.

13. Gupta S, Jiang M and Pernis AB: IFN-alpha activates Stat6 and leads to the formation of Stat2:Stat6 complexes in B cells. J Immunol 163: 3834-3841, 1999.

14. Dickensheets H, Vazquez N, Sheikh F, Gingras S, Murray PJ, Ryan JJ and Donnelly RP: Suppressor of cytokine signaling-1 is an IL-4-inducible gene in macrophages and feedback inhibits IL-4 signaling. Genes Immun 8: 21-27, 2007.

15. Li Y, Deuring J, Peppelenbosch MP, Kuipers EJ, de Haar C and van der Woude CJ: STAT1, STAT6 and adenosine 3',5'-cyclic monophosphate (cAMP) signaling drive SOCS3 expression in inactive ulcerative colitis. Mol Med 18: 1412-1419, 2012.

16. Hanson EM, Dickensheets H, Qu CK, Donnelly RP and Keegan AD: Regulation of the dephosphorylation of Stat6. Participation of Tyr-713 in the interleukin-4 receptor alpha, the tyrosine phosphatase SHP-1, and the proteasome. J Biol Chem 278: 3903-3911, 2003.

17. Lu X, Malumbres R, Shields B, Jiang X, Sarosiek KA, Natkunam Y, Tiganis T and Lossos IS: PTP1B is a negative regulator of interleukin 4-induced STAT6 signaling. Blood 112: 4098-4108, 2008.

18. Chen W, Daines MO and Hershey GK: Methylation of STAT6 modulates STAT6 phosphorylation, nuclear translocation, and DNA-binding activity. J Immunol 172: 6744-6750, 2004.

19. Di Stefano AB, Iovino F, Lombardo Y, Eterno V, Höger T, Dieli F, Stassi G and Todaro M: Survivin is regulated by interleukin-4 in colon cancer stem cells. J Cell Physiol 225: 555-561, 2010.

20. Huang F, Wang D, Yao YL and Wang M: PDGF signaling in cancer progression. Int J Clin Exp Med 10: 9918-9929, 2017.

21. Patel BK, Wang LM, Lee CC, Taylor WG, Pierce JH and LaRochelle WJ: Stat6 and Jak1 are common elements in platelet-derived growth factor and interleukin-4 signal transduction pathways in NIH 3T3 fibroblasts. J Biol Chem 271: 22175-22182, 1996 .

22. Ingram JL, Antao-Menezes A, Mangum JB, Lyght O, Lee PJ, Elias JA and Bonner JC: Opposing actions of Stat1 and Stat6 on IL-13-induced up-regulation of early growth response-1 and platelet-derived growth factor ligands in pulmonary fibroblasts. J Immunol 177: 4141-4148, 2006.

23. Jess T, Rungoe $\mathrm{C}$ and Peyrin-Biroulet L: Risk of colorectal cancer in patients with ulcerative colitis: A meta-analysis of population-based cohort studies. Clin Gastroenterol Hepatol 10: 639-645, 2012

24. von Roon AC, Reese G, Teare J, Constantinides V, Darzi AW and Tekkis PP: The risk of cancer in patients with Crohn's disease. Dis Colon Rectum 50: 839-855, 2007.

25. Rosen MJ, Frey MR, Washington MK, Chaturvedi R, Kuhnhein LA, Matta P, Revetta FL, Wilson KT and Polk DB: STAT6 activation in ulcerative colitis: A new target for prevention of IL-13-induced colon epithelial cell dysfunction. Inflamm Bowel Dis 17: 2224-2234, 2011.

26. Wang CG, Ye YJ, Yuan J, Liu FF, Zhang H and Wang S: EZH2 and STAT6 expression profiles are correlated with colorectal cancer stage and prognosis. World J Gastroenterol 16: 2421-2427, 2010.

27. Tosolini M, Kirilovsky A, Mlecnik B, Fredriksen T, Mauger S, Bindea $G$, Berger A, Bruneval P, Fridman WH, Pagès $F$ and Galon J: Clinical impact of different classes of infiltrating T cytotoxic and helper cells (Th1, th2, treg, th17) in patients with colorectal cancer. Cancer Res 71: 1263-1271, 2011.

28. Formentini A, Braun P, Fricke H, Link KH, Henne-Bruns D and Kornmann M: Expression of interleukin-4 and interleukin-13 and their receptors in colorectal cancer. Int J Colorectal Dis 27: 1369-1376, 2012

29. Rosen MJ, Chaturvedi R, Washington MK, Kuhnhein LA, Moore PD, Coggeshall SS, McDonough EM, Weitkamp JH, Singh AB, Coburn LA, et al: STAT6 deficiency ameliorates severity of oxazolone colitis by decreasing expression of claudin-2 and Th2-inducing cytokines. J Immunol 190: 1849-1858, 2013.

30. Hoving JC, Kirstein F, Nieuwenhuizen NE, Fick LC, Hobeika E, Reth $\mathrm{M}$ and Brombacher F: B Cells that produce immunoglobulin E mediate Colitis in BALB/c mice. Gastroenterology 142: 96-108, 2012 
31. Buzza MS, Johnson TA, Conway GD, Martin EW Mukhopadhyay S, Shea-Donohue T and Antalis TM: Inflammatory cytokines down-regulate the barrier-protective prostasin-matriptase proteolytic cascade early in experimental colitis. J Biol Chem 292: 10801-10812, 2017.

32. Shajib MS, Wang HQ, Kim JJ, Sunjic I, Ghia JE, Denou E, Collins M, Denburg JA and Khan WI: Interleukin 13 and Serotonin: Linking the immune and endocrine systems in murine models of intestinal inflammation. PLoS One 8: e72774, 2013.

33. Leon-Cabrera SA, Molina-Guzman E, Delgado-Ramirez YG, Vázquez-Sandoval A, Ledesma-Soto Y, Pérez-Plasencia CG, Chirino YI, Delgado-Buenrostro NL, Rodríguez-Sosa M, Vaca-Paniagua F, et al: Lack of STAT6 attenuates inflammation and drives protection against early steps of colitis-associated colon cancer. Cancer Immunol Res 5: 385-396, 2017.

34. Jayakumar A and Bothwell ALM: Stat6 promotes intestinal tumorigenesis in a mouse model of adenomatous polyposis by expansion of MDSCs and inhibition of cytotoxic CD8 response. Neoplasia 19: 595-605, 2017

35. De Oliveira T, Ramakrishnan M, Diamanti MA, Ziegler PK, Brombacher F and Greten FR: Loss of Stat6 affects chromatin condensation in intestinal epithelial cells causing diverse outcome in murine models of inflammation-associated and sporadic colon carcinogenesis. Oncogene 38: 1787-1801, 2019.

36. Wick EC, LeBlanc RE, Ortega G, Robinson C, Platz E, Pardoll DM, Iacobuzio-Donahue C and Sears CL: Shift from pStat6 to pStat3 predominance is associated with inflammatory bowel disease-associated dysplasia. Inflamm Bowel Dis 18: 1267-1274, 2012.

37. Cao H, Zhang J, Liu H, Wan L, Zhang H, Huang Q, Xu E and Lai M: IL-13/STAT6 signaling plays a critical role in the epithelial-mesenchymal transition of colorectal cancer cells. Oncotarget 7: 61183-61198, 2016.

38. Jiang L, Cheng Q, Zhang B and Zhang M: IL-13 induces the expression of $11 \beta \mathrm{HSD} 2$ in IL-13R $\alpha 2$ dependent manner and promotes the malignancy of colorectal cancer. Am J Transl Res 8: 1064-1072, 2016.

39. Barderas R, Bartolome RA, Fernandez-Acenero MJ, Torres S and Casal JI: High expression of IL-13 receptor $\alpha 2$ in colorectal cancer is associated with invasion, liver metastasis, and poor prognosis. Cancer Res 72: 2780-2790, 2012.

40. Chen J, Gong C, Mao H, Li Z, Fang Z, Chen Q, Lin M, Jiang X, $\mathrm{Hu} \mathrm{Y}$, Wang W, et al: E2F1/SP3/STAT6 axis is required for IL-4-induced epithelial-mesenchymal transition of colorectal cancer cells. Int J Oncol 53: 567-578, 2018

41. Todaro M, Lombardo Y, Francipane MG, Alea MP, Cammareri P, Iovino F, Di Stefano AB, Di Bernardo C, Agrusa A, Condorelli $\mathrm{G}$, et al: Apoptosis resistance in epithelial tumors is mediated by tumor-cell-derived interleukin-4. Cell Death Differ 15: 762-772, 2008.

42. Liu H, Antony S, Roy K, Juhasz A, Wu Y, Lu J, Meitzler JL, Jiang G, Polley E and Doroshow JH: Interleukin-4 and interleukin-13 increase NADPH oxidase 1-related proliferation of human colon cancer cells. Oncotarget 8: 38113-38135, 2017.

43. Kanai T, Watanabe M, Hayashi A, Nakazawa A, Yajima T, Okazawa A, Yamazaki M, Ishii H and Hibi T: Regulatory effect of interleukin-4 and interleukin-13 on colon cancer cell adhesion. Br J Cancer 82: 1717-1723, 2000.

44. Binnemars-Postma K, Bansal R, Storm G and Prakash J: Targeting the Stat6 pathway in tumor-associated macrophages reduces tumor growth and metastatic niche formation in breast cancer. FASEB J 32: 969-978, 2018.

45. Linde N, Lederle W, Depner S, van Rooijen N, Gutschalk CM and Mueller MM: Vascular endothelial growth factor-induced skin carcinogenesis depends on recruitment and alternative activation of macrophages. J Pathol 227: 17-28, 2012.

46. Ostrand-Rosenberg S, Sinha P, Clements V, Dissanayake SI, Miller S, Davis C and Danna E: Signal transducer and activator of transcription 6 (Stat6) and CD1: Inhibitors of immunosurveillance against primary tumors and metastatic disease. Cancer Immunol Immunother 53: 86-91, 2004.

47. Conticello C, Pedini F, Zeuner A, Patti M, Zerilli M, Stassi G, Messina A, Peschle C and De Maria R: IL-4 protects tumor cells from anti-CD95 and chemotherapeutic agents via up-regulation of antiapoptotic proteins. J Immunol 172: 5467-5477, 2004.

48. Francipane MG, Alea MP, Lombardo Y, Todaro M, Medema JP and Stassi G: Crucial role of interleukin-4 in the survival of colon cancer stem cells. Cancer Res 68: 4022-4025, 2008.

49. Li BH, Yang XZ, Li PD, Yuan Q, Liu XH, Yuan J and Zhang WJ: IL-4/Stat 6 activities correlate with apoptosis and metastasis in colon cancer cells. Biochem Biophys Res Commun 369: 554-560, 2008.
50. Li BH, Xu SB, Li F, Zou XG, Saimaiti A, Simayi D, Wang YH, Zhang Y, Yuan J and Zhang WJ: Stat6 activity-related Th2 cytokine profile and tumor growth advantage of human colorectal cancer cells in vitro and in vivo. Cell Signal 24: 718-725, 2012.

51. Volonte A, Di Tomaso T, Spinelli M, Todaro M, Sanvito F, Albarello L, Bissolati M, Ghirardelli L, Orsenigo E, Ferrone S, et al: Cancer-initiating cells from colorectal cancer patients escape from $\mathrm{T}$ cell-mediated immunosurveillance in vitro through membrane-bound IL-4. J Immunol 192: 523-532, 2014.

52. Cosin-Roger J, Ortiz-Masia D, Calatayud S, Hernandez C, Esplugues JV and Barrachina MD: The activation of Wnt signaling by a STAT6-dependent macrophage phenotype promotes mucosal repair in murine IBD. Mucosal Immunol 9: 986-998, 2016

53. Mendoza-Rodriguez MG, Sanchez-Barrera CA, Callejas BE, García-Castillo V, Beristain-Terrazas DL, Delgado-Buenrostro NL, Chirino YI, León-Cabrera SA, Rodríguez-Sosa M, Gutierrez-Cirlos EB, et al: Use of STAT6 Phosphorylation inhibitor and trimethylglycine as new adjuvant therapies for 5-fluorouracil in colitis-associated tumorigenesis. Int J Mol Sci 21: pii: E2130, 2020.

54. Nappo G, Handle F, Santer FR, McNeill RV, Seed RI, Collins AT, Morrone G, Culig Z, Maitland NJ and Erb HHH: The immunosuppressive cytokine interleukin- 4 increases the clonogenic potential of prostate stem-like cells by activation of STAT6 signalling. Oncogenesis 6: e342, 2017.

55. Xu K, Tao W and Su Z: Propofol prevents IL-13-induced epithelial-mesenchymal transition in human colorectal cancer cells. Cell Biol Int 42: 985-993, 2018.

56. Healey GD, Lockridge JA, Zinnen S, Hopkin JM, Richards I and Walker W: Development of pre-clinical models for evaluating the therapeutic potential of candidate siRNA targeting STAT6. PLoS One 9: e90338, 2014

57. Bartolome RA, Jaen M and Casal JI: An IL13R $\alpha 2$ peptide exhibits therapeutic activity against metastatic colorectal cancer. Br J Cancer 119: 940-949, 2018.

58. Gharib AF, Shalaby SM, Raafat N, Fawzy WMS and Abdel Hakim NH: Assessment of neutralizing interleukin-4 effect on CD133 gene expression in colon cancer cell line. Cytokine 97: 66-72, 2017.

59. Todaro M, Perez Alea M, Scopelliti A, Medema JP and Stassi G: IL-4-mediated drug resistance in colon cancer stem cells. Cell Cycle 7: 309-313, 2008

60. Todaro M, Alea MP, Di Stefano AB, Cammareri P, Vermeulen L, Iovino F, Tripodo C, Russo A, Gulotta G, Medema JP and Stassi G: Colon cancer stem cells dictate tumor growth and resist cell death by production of interleukin-4. Cell Stem Cell 1: 389-402, 2007.

61. Rivankar S: An overview of doxorubicin formulations in cancer therapy. J Cancer Res Ther 10: 853-858, 2014.

62. Yang CY, Liu HW, Tsai YC, Tseng JY, Liang SC, Chen CY, Lian WN, Wei MC, Lu M, Lu RH, et al: Interleukin-4 receptor-targeted liposomal doxorubicin as a model for enhancing cellular uptake and antitumor efficacy in murine colorectal cancer. Cancer Biol Ther 16: 1641-1650, 2015.

63. O'Shea JJ, Kontzias A, Yamaoka K, Tanaka Y and Laurence A: Janus kinase inhibitors in autoimmune diseases. Ann Rheum Dis 72 (Suppl 2): ii111-ii115, 2013.

64. Regenbogen T, Chen L, Trinkaus K, Wang-Gillam A, Tan BR, Amin M, Pedersen KS, Park H, Suresh R, Lim KH, et al: Pacritinib to inhibit JAK/STAT signaling in refractory metastatic colon and rectal cancer. J Gastrointest Oncol 8: 985-989, 2017.

65. Pardanani A and Tefferi A: How I treat myelofibrosis after failure of JAK inhibitors. Blood 132: 492-500, 2018.

66. Fogelman D, Cubillo A, Garcia-Alfonso P, Mirón MLL, Nemunaitis J, Flora D, Borg C, Mineur L, Vieitez JM, Cohn A, et al: Randomized, double-blind, phase two study of ruxolitinib plus regorafenib in patients with relapsed/refractory metastatic colorectal cancer. Cancer Med 7: 5382-5393, 2018

67. Shimaoka H, Takeno S, Maki K, Sasaki T, Hasegawa S and Yamashita Y: A cy tokine signal inhibitor for rheumatoid arthritis enhances cancer metastasis via depletion of NK cells in an experimental lung metastasis mouse model of colon cancer. Oncol Lett 14: 3019-3027, 2017.

This work is licensed under a Creative Commons Attribution-NonCommercial-NoDerivatives 4.0 International (CC BY-NC-ND 4.0) License. 